www. revis tad yo. com

\title{
Análisis comparativo del problema de conformación de lotes con ruteo en la preparación de pedidos respecto al HVRP (Heterogeneous Vehicle Routing Problem)
}

Comparative analysis of order batching and routing problem in the picking regarding classical HVRP (heterogeneous vehicle routing problem)

\author{
Rodrigo Gómez¹, Alexander Correa ${ }^{1}$, Jesús Muñuzuri² y Pablo Cortes².
}

1Departamento Ingeniería de la Organización. Universidad Nacional de Colombia, Sede Medellín. Carrera 80 No 65-223 Medellín, Colombia. ${ }^{2}$ Dpto. de Organización Industrial y Gestión de Empresas II. ETSI. Universidad de Sevilla. Camino de los Descubrimientos, s/n. Isla de la Cartuja, 41092, Sevilla, España.

ragomez@elpoli.edu.co, alcorrea@unal.edu.co,munuzuri@etsi.us.es,pca@us.es

Fecha de recepción: 4-12-2014

Fecha de aceptación: 11-06-2016

Resumen: Este artículo tiene como objetivo comparar la conformación de lotes con ruteo, en la preparación de pedidos respecto al problema HVRP (Heterogeneous Vehicle Routing Problem) basándose en la utilización de una metodología de la revisión sistemática de la literatura. Del análisis comparativo se identifica la necesidad de realizar modificaciones radicales e incluir nuevos componentes al problema HVRP, para modelar la conformación de lotes con ruteo de mínimo tiempo, en la preparación de pedidos, considerando $\mathrm{K}$ equipos de manejo de materiales (EMM) heterogéneos, $\mathrm{n}$ productos, $\mathrm{m}$ posiciones de almacenamiento, la disponibilidad del inventario y demás restricciones asociadas a la operación.

Palabras claves: Conformación de lotes, ruteo, preparación de pedidos, centros de distribución y HVRP (Heterogeneous Vehicle Routing Problem).

\begin{abstract}
This paper aims to compare the order batching and routing problem(OBRP) regarding heterogeneous vehicle routing problem (HVRP), in order to identify whether there are any differences and similarities between these ones. The OBRP consist in generating product groups, which are collected from storage locations using material specific handling equipment. Each product group(or batch) is matched to a route, which states the sequences to pick the products in the shortest time possible. On the other hand, HVRP is a variant of the Vehicle Routing Problem(VRP), in which customers are served by a heterogeneous fleet of vehicles with various capacities, in order to delivery products in a distribution network at the lowest possible cost. Additionally, in the related literature were not identified HVRP papers that tackled order batching and routing problem (OBRP), but they were focused primarily in transportation and distribution process. Therefore, it was detected a gap in the state of the art. The comparation analysis was developed using a variation of the methodology called Systematic Literature Review (SLR), which was based on analysis of papers. This methodology was implemented eight stages, the most important of which are as follows: i) formulating the research questions and evaluation criteria (stage 2), ii) inclusion and exclusion criteria (stage 3), iii) results of systematic review (stage 6), iv) comparative analysis between OBRP and HVRP based on set evaluation criteria (stage 7) and v) conclusions and research opportunities (stage 8). The main findings of this paper were as follow: First, order batching was not modeled in HVRP, hence relevance of this gap. Second, in order batching and routing problem is necessary to represent $\mathrm{K}$ heterogeneous MHE with different speed travels, load capacities and lift heights. In HVRP papers the heterogeneity is only caused by vehicles in different load capacities. Third, a constraint among $\mathrm{n}$ products, $\mathrm{m}$ storage locations and $\mathrm{K}$ heterogeneous $\mathrm{EMH}$ should be implemented to ensure the feasibility of solutions of OBRP. This constraint is raised, since any MHE are not able to pick some products from storage locations, due to theirs technical characteristics. In addition, none of HVRP papers represented this constraint. Fourth, setup time and handling time were not modeled in reviewed HVRP papers, since these times were not as significant in transportation and distribution routes. Therefore, these times should be included in HVRP to represent OBRP. Fifth, available of inventory were not considered in HVRP papers, since this condition was not important in the modeled process. It should be noted that this condition is critical in OBRP, since only can be picked products with available inventory in Distribution Centre (DC). Based on findings, it was detected a significant gap in the state of the art related to the formulation and solution a minimum time OBRP considering $\mathrm{n}$ products, $\mathrm{m}$ storage locations and $\mathrm{K}$ heterogeneous $\mathrm{MHE}$ and described constraint. Therefore, this approach, not only it will fill this gap, but also contribute to knowledge in OBRP. In addition, this paper it will be one of the first to analyze HVRP in warehouse and DC.
\end{abstract}

Keywords: Order batching, routing, picking, Distribution Centre and HVRP (Heterogeneous Vehicle Routing Problem). 


\section{Introducción}

La preparación de pedidos es la operación más importante de un Centro de Distribución(CEDI), ya que se encarga de recoger los productos solicitados por los clientes, en las condiciones y tiempo pactado (Bartholdi y Hackman, 2011 ; Henn y Schmid, 2013). Esta operación también tiene importancia desde la perspectiva económica, ya que representa entre el 50 y $65 \%$ de los costes operativos en un CEDI manual (Min, 2009).

En la preparación de pedidos, la conformación de lotes con ruteo ha sido considerado como un problema táctico y operativo, que permite formar grupos de productos y establecer secuencias de recogida de mínimo tiempo o tardanza, que permitan satisfacer adecuadamente los requerimientos de los clientes a niveles de eficiencia adecuados (Kulak et al., 2012; Henn, 2012; Henn y Schmid, 2013; Matusiak et al., 2014). Adicionalmente, este problema ha sido clasificado en la literatura como NP-Hard, debido a las restricciones que se presentan entre los productos y posiciones de almacenamiento, y la complejidad de resolver problemas grandes (más de 40 posiciones de almacenamiento) debido a las limitaciones de capacidad computacional. Por esta razón, en la literatura para problemas de conformación de lotes con ruteo grandes, se han utilizado metaheurísticos, los cuales han generado buenas soluciones (Henn, 2010; Henn, 2012; Kulak et al., 2012).

La conformación de lotes con ruteo, consiste en generar grupos con los $n$ productos $i^{€}$ OC (Órdenes de los clientes), los cuales, deben ser recogidos de $\mathrm{m}$ posi-ciones de almacenamiento en el menor tiempo total usando equipos de manejo de materiales (EMM) o personas (Henn, 2012; Chen et al., 2014). A continu-ación, se representan los componentes descritos de la conformación de lotes con ruteo (Figura 1).

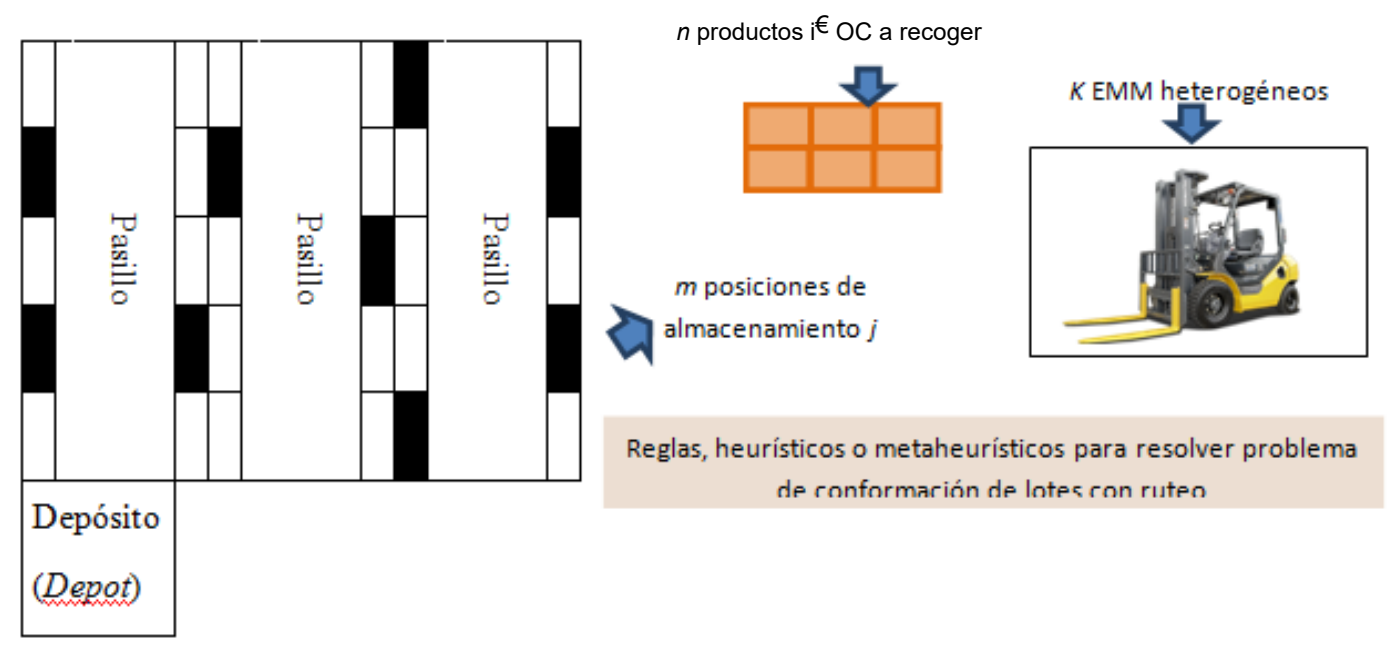

Figura 1. Componentes de la conformación de lotes con ruteo, en la preparación de pedidos.

De otra parte, en los problemas de conformación de lotes con ruteo en la preparación de pedidos publicados en la literatura, se han modelado los equipos de manejo de materiales (EMM) desde tres enfoques. En el primer enfoque, se modelan los EMM de capacidad infinita, lo cual, reduce la complejidad del problema (Daniels et al., 1998; De Koster et al., 1999), (Manzini et al., 2005; Tsai et al., 2008; Dharmapriya y Kulatunga, 2011). En el segundo enfoque, los problemas revisados representaron un único equipo de manejo de materiales de capacidad fija, ya que se conformaba un lote con ruteo a la vez (Ho et al., 2008; Henn, 2010; Henn y Wäsher, 2012; Kulak et al., 2012; Mastusiak et al., 2014). En tanto, el tercer enfoque, se modelan $\mathrm{K}$ equipos homogéneos (igual capacidad, velocidad y manejo de productos en altura) o K preparadores hu- manos, los cuales poseen limitaciones para manipular productos en altura (Albareda et al., 2009; Rubrico et al., 2011; Henn, 2012). A partir de estos hallazgos, se detecta que no se han publicado artículos que modelen K EMM heterogéneos en el problema de conformación de lotes. Este vacío puede limitar la implementación del problema en el ámbito empresarial, donde suelen existir K EMM heterogéneos, que permiten ejecutar la preparación de pedidos, según el tipo de producto a recoger y el nivel de altura de la posición de almacenamiento a visitar.

El modelado de $\mathrm{K}$ equipos heterogéneos en la configuración de rutas de mínimo tiempo o costo, han sido abordado en la literatura a través de un problema denominado HVRP (Heterogeneous Vehicle Routing Problem) (Prins, 2012; De la Cruz et al., 2013; Le- 
ung et al., 2013). En el ámbito logístico, el HVRP ha sido implementado en procesos de transporte y distribución, con el objetivo de diseñar rutas de mínimo tiempo utilizando camiones heterogéneos (diferente capacidad de carga). De la revisión de la literatura realizada, no se detectaron aplicaciones o adaptaciones del problema HVRP, en la preparación de pedidos $u$ otras operaciones del Centro de Distribución (CEDI) como el acomodo, las cuales, suele utilizar $\mathrm{K}$ equipos heterogéneos para ejecutar sus actividades. Adicionalmente, debe resaltarse que en la literatura científica, no fueron detectados artículos que estudiaran teóricamente la comparación de estos problemas.

A partir de este hallazgo, se detecta una oportunidad de investigación relacionada con el desarrollo de un análisis comparativo del problema de conformación de lotes con ruteo, en la preparación de pedidos respecto a un problema HVRP, con el fin de determinar su diferencias, similaridades e identificación de componentes que pueden ser adaptadas para modelar los $\mathrm{K}$ equipos heterogéneos, en la operación del CEDI en estudio. Para elaborar el análisis comparativo, se desarrolla una adaptación del método de revisión sistemática de la literatura planteada por Kitchenham (2004). De esta manera, se pretende realizar un análisis detallado, que permita estudiar apropiadamente los problemas en estudio.
El resto del artículo está organizado como sigue: En la sección 2 es desarrollada la metodología adaptada para la revisión sistemática de la literatura (SLR). En la sección 3 , se presentan los resultados de la búsqueda de la literatura científica. En la sección 4, se presenta una síntesis del análisis comparativo entre el problema de conformación de lotes con ruteo, en la preparación de pedidos y el HVRP. Finalmente, en la sección 5, se establecen las líneas futuras de investigación y las conclusiones.

\section{Metodología de la revisión sis- temática de la literatura(SLR)}

El análisis de los antecedentes y el estado del arte, se realiza con una adaptación a la metodología de la revisión sistemática de la literatura (SLR Systematic Literature Review, en inglés). La metodología SLR adaptada, se diferencia a la original, ya que se incluyen dos etapas adicionales denominadas: criterios de evaluación y comparación de la conformación de lotes con ruteo respecto al HVRP. A continuación, se presentan las ocho etapas de la metodología SLR adaptada (Figura 2).

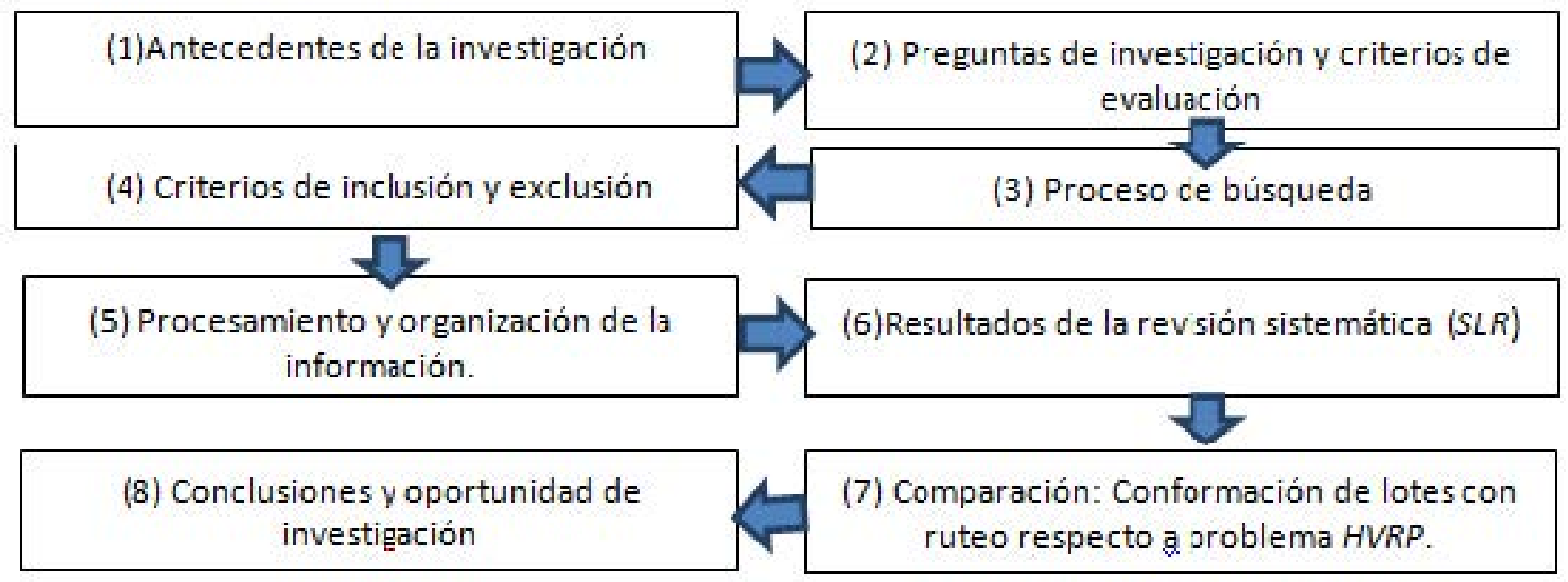

Figura 2. Etapas metodología SLR adaptada. 
La metodología SLR adaptada (Figura 2), inicia con una descripción de los antecedentes de la investigación (etapa 1), la formulación de tres preguntas y establecimiento de los criterios de evaluación, los cuales orientan la búsqueda de la información y el desarrollo del análisis comparativo planteado (etapa 2). Posteriormente, se estructura el proceso de búsqueda, en el cual, se definen las palabras claves, ecuaciones de búsqueda, selección de bases de datos a consultar, criterios de inclusión y exclusión de artículos científicos a analizar (etapa 3) y (etapa 4). Una vez se recolectan y revisan los artículos, se procede a su procesamiento, con el fin de organizar la información obtenida, y determinar si es necesario complementar la búsqueda (etapa 5).

A partir de la información organizada, se responde las preguntas de investigación, y se presentan los resultados del SLR por medio de: i) una tabla del síntesis del estado del arte (autor y evaluación de criterios) y ii) gráficos que permiten resumir los resultados principales de la evaluación de criterios establecidos en el SLR (etapa 6). Posteriormente, se realiza el análisis comparativo del de la conformación de lotes con ruteo, en la preparación de pedidos respecto al problema HVRP clásico, que permite establecer las diferencias y similitudes. Finalmente, se establecen las oportunidades de investigación y las conclusiones obtenidas del SLR(etapa 8).

A continuación, se presentan las etapas de la uno a la cuatro planteadas en la metodología SLR.

\section{- $\quad$ Antecedentes de la investigación (etapa 1):}

EI HVRP (Heterogeneous Vehicle Routing Problem) es una variante del problema VRP (Vehicle RoutingProblem), que tiene como objetivo diseñar rutas de mínimo coste para atender los requerimientos de los clientes y establecer la composición de flotas heterogéneas de vehículos de diferentes capacidades de carga, costes fijos y variables (Choi y Tcha, 2007; Imran et al., 2009; Prins, 2009; Prins, 2012; Subramanian et al. ,2012; Penna et al. ,2013).

De un análisis exploratorio de la literatura, no se detectaron artículos que modelaran el problema HVRP, en la conformación de lotes con ruteo o la configuración de rutas de mínimo tiempo o tardanza, en la preparación de pedidos; sino que los autores, se enfocaban principalmente, en el modelado del transporte, distribución u otros procesos logísticos genéricos, que no consideraban las variables y restricciones de esta operación del CEDI.

Adicionalmente, en la revisión del estado del arte del problema de conformación de lotes con ruteo, en la preparación de pedidos, no se identificaron artículos que modelaran $\mathrm{K}$ EMM heterogéneos, sino que este recurso se modelaba desde tres enfoques: i) EMM de capacidad infinita, ii) un EMM de capacidad fija, ya que se conformaba de a un lote con ruteo a la vez, y iii) $\mathrm{K}$ EMM homogéneos o preparadores humanos de pedidos. De esta manera, se detectó la ausencia de los enfoques que modelaran $\mathrm{K}$ equipos heterogéneos en este problema en la preparación de pedidos.

Debido a la ausencia de artículos que modelaran $\mathrm{K}$ EMM heterogéneos en el problema de conformación de lotes con ruteo, en la preparación de pedidos, se identificó la necesidad de realizar un análisis comparativo respecto a los problemas HVRP publicados en la literatura, con el propósito de evaluar sus diferencias y semejanzas.

- $\quad$ Formulación de preguntas de investigación y criterios de evaluación (etapa 2)

La presente SLR está basada en las siguientes preguntas de investigación $(\mathrm{PI})$ :

o PI1: ¿Existen diferencias entre la conformación de lotes con ruteo, en la preparación de pedidos y el problema HVRP?

- PI2: ¿En caso de presentarse diferencias entre los problemas comparados, cuáles son las características y magnitudes de estas?

o PI3: ¿En caso de existir discrepancias entre los problemas evaluados, se hace necesario formular un nuevo problema de conformación de lotes con ruteo, en la preparación de pedidos, o pueden realizarse sencillas adaptaciones a alguno de los artículos que representan el problema HVRP?

Para facilitar el desarrollo del análisis comparativo y obtener información para las preguntas de investigación, el problema de conformación de lotes con ruteo, es dividido en los siguientes criterios, los cuales, se evalúan para cada uno de los artículos HVRP (Figura 3). 

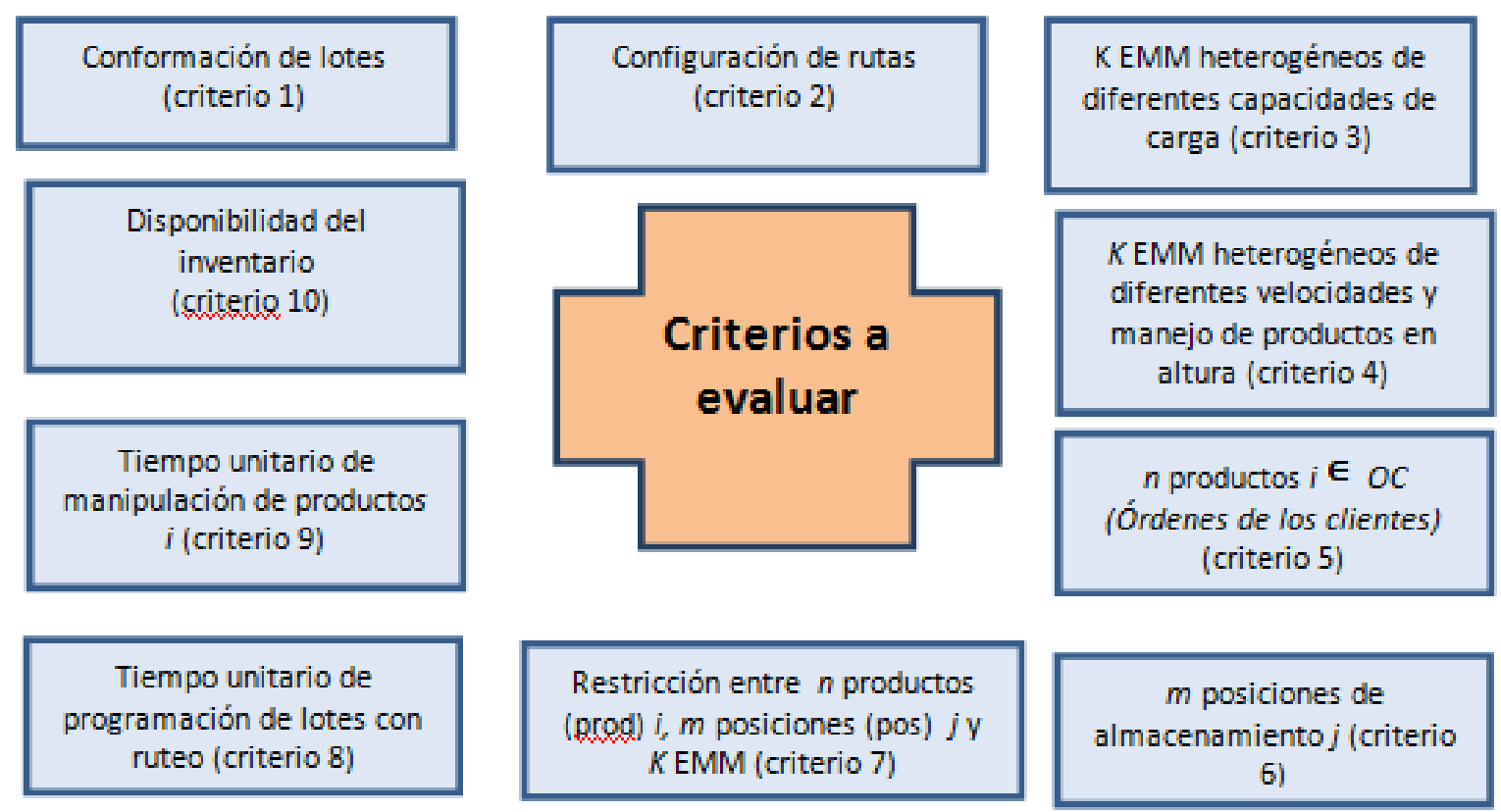

Figura 3. Criterios a evaluar de la conformación de lotes con ruteo, en la preparación de pedidos.

A continuación, se describe el proceso de búsqueda (etapa 3) y los criterios de inclusión y exclusión de los artículos) (etapa 4) de la metodología del SLR adaptada(Tabla 1).

Tabla 1. Proceso de búsqueda, criterios de inclusión y exclusión en la metodología SLR.

\section{Proceso de búsqueda(Etapa 3)}

- Se consultan bases de datos con artículos científicos indexados como: Science Direct, Emerald, Taylor and Francis, Springer, SCIELO y REDALYC

- Se utiliza metabuscadores como SCOPUS y GOOGLE SCHOLAR.

- $\quad$ Base de datos IEEE con artículos de conferencias.

- Se detectaron 50 artículos entre el año 1999 y 2014, de los cuales, se seleccionaron 19 que cumplieron con los criterios de inclusión. Los artículos seleccionados para el SLR, se almacenan y gestionan con el administrador de referencias bibliográficas ZOTERO $4.0{ }^{\circledR}$

\section{Criterios de inclusión(Etapa 4)}

o $\quad$ Artículos de investigación con formulación algebraica del problema y validación experimental de los resultados.

o $\quad$ Artículos de conferencias registrados en la IEEE con formulación algebraica y validación experimental de los resultados.

Criterios de exclusión(Etapa 4)

- Artículos que desarrollen casos de estudio, que no consideren formulación algebraica y metodología de modelamiento del problema.

- Capítulos de libro. 
Finalmente, la etapa cinco no será presentada en este artículo, ya que consiste en procesar y organizar la información, que permite realizar la comparación planteada.

\section{Resultados de la metodología SLR}

Los resultados de la metodología SLR, se presentan en dos partes. En la primera parte se elabora una tabla que sintetiza los resultados del análisis de los diez criterios de evaluación en los 19 artículos detectados para el problema HVRP (Figura 2). En la síntesis, para cada autor se registra, si cumplió o no, con el criterio analizado (Tabla 2). En tanto, en la segunda parte, se realiza un análisis detallado de las contribuciones en cada uno de los 10 criterios evaluados para la conformación de lotes con ruteo en la preparación de pedidos y el HVRP. Para la calificación, se utilizó el símbolo X, para indicar que el artículo si cumplió con el criterio evaluado, y vacío, para registrar que no.

Tabla 2. Síntesis de análisis de criterios de evaluación en el problema HVRP

\begin{tabular}{|c|c|c|c|c|c|c|c|c|c|c|}
\hline Autores & C1 & $\mathrm{C} 2$ & C3 & C4 & C5 & C6 & C7 & $\mathrm{C} 8$ & C9 & C10 \\
\hline Gendreau et al.,(1999) & & $x$ & $x$ & & & $x$ & $x$ & & & \\
\hline Prins(2002) & & $x$ & $x$ & & & $x$ & & $x$ & & \\
\hline Choi y Tcha(2007) & & $x$ & $x$ & & & $x$ & & & & \\
\hline Prado y Lopes(2007) & & $x$ & $x$ & & & $x$ & $x$ & & & \\
\hline Imran et al.,(2009) & & $x$ & $x$ & & & $x$ & & & & \\
\hline Brandao et al., (2009) & & $x$ & $x$ & & & $x$ & & & & \\
\hline Prins(2009) & & $x$ & $x$ & & & & & & & \\
\hline Rekersbrink et al.,(2009) & & $x$ & $x$ & & $x$ & & & & & \\
\hline Ceschia et al.,(2010) & & $x$ & $x$ & & & $\mathrm{x}$ & & $\mathrm{x}$ & & \\
\hline Euchi y Chabchoub(2010) & & $\mathrm{x}$ & $\mathrm{x}$ & & & $\mathrm{x}$ & $x$ & & & \\
\hline Ozfirat y Ozkarahan(2010) & & $\mathrm{x}$ & $\mathrm{x}$ & & & $x$ & & & & \\
\hline Brandao (2011) & & $x$ & $x$ & & & $x$ & & & & \\
\hline Li et al.,(2012) & & $x$ & $x$ & & & $x$ & $x$ & & & \\
\hline Duhamel et al.,(2012) & & $x$ & $x$ & & & $x$ & & & & \\
\hline Subramanian et al.,(2012) & & $x$ & $x$ & & & $x$ & & & & \\
\hline De la Cruz et al., (2013) & & $x$ & $x$ & & $x$ & $x$ & & & & \\
\hline Leung et al.,(2013) & & $x$ & $x$ & & $x$ & $x$ & $x$ & & & \\
\hline Kritikos y loannou(2013) & & $x$ & $x$ & & & $x$ & $x$ & & & \\
\hline Bjelić et al.,(2013) & & $\mathrm{x}$ & $\mathrm{x}$ & & & $\mathrm{x}$ & & & & \\
\hline
\end{tabular}


De los resultados obtenidos del SLR (Tabla 2), no se identificaron artículos del problema HVRP, que implementaran integralmente los 10 criterios evaluados del problema de conformación de lotes con ruteo, en la preparación de pedidos. Del análisis, se detecta que la configuración de rutas (criterio 2), el modelamiento de $K$ vehículos heterogéneos de diferentes capacidades de carga (criterio 3) y la representación de $\mathrm{M}$ nodos (criterio 6) fueron los únicos criterios del problema HVRP que fueron apropiadamente abordados en la conformación de lotes con ruteo. Los otros siete criterios analizados no fueron abordados directamente en el problema HVRP, o fueron modelados de forma parcial, lo cual, permitió inferir que no se cumplían.

Para ampliar los resultados del análisis comparativo, a continuación, se representan y discuten los principales hallazgos detectados para los diferentes diez criterios evaluados (Figura 4).

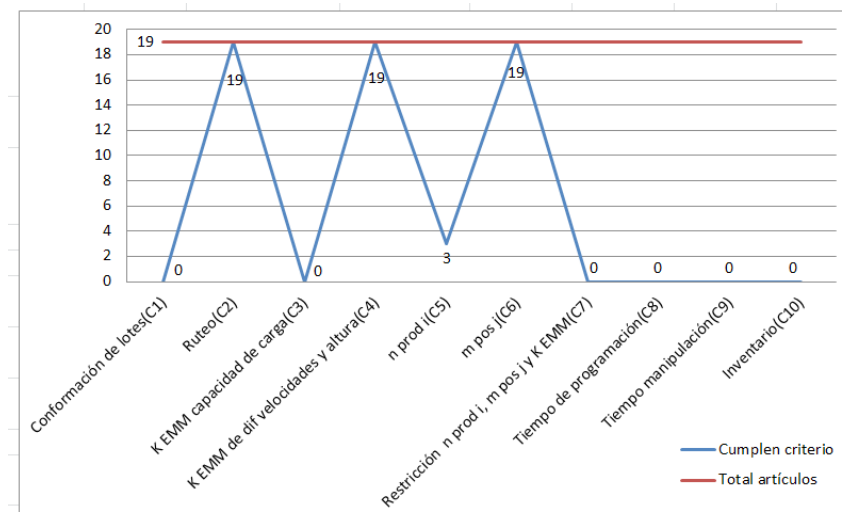

Figura 4. Representación de resultados de evaluación de los criterios de la conformación de lotes con ruteo respecto al problema HVRP.

\section{- $\quad$ Conformación de lotes (criterio 1) y ruteo (criterio 2)}

Respecto al criterio uno, ninguno de los 19 artículos evaluados modeló la conformación de lotes, en el problema HVRP, sino que los artículos se enfocaban en la configuración de rutas de mínimo coste total(o tiempo total) en procesos de transporte, distribución u otros procesos logísticos combinatorios diferentes a la preparación de pedidos. En los artículos evaluados, el diseño de rutas consistía en la generación y evaluación de combinaciones factibles de $\mathrm{m}$ clientes (nodos) y K vehículos de diferentes capacidades (flota heterogénea), que permitían obtener secuencias rutas de mínimo coste.

La mayoría de los artículos evaluados tenían como objetivo configurar rutas de mínimo coste total (fijos y variables), ya que los vehículos empleados recorrían distancias (kilómetros, km) considerables entre los nodos de una red geográfica, con el fin de satisfacer las necesidades de los clientes. En el contexto de la preparación de pedidos, la minimización de costes no es tan importante, como lo la obtención de tiempos mínimos de la operación, ya que la prioridad de la recogida de las órdenes es atender los requerimientos de los clientes en las condiciones logísticas pactadas. Adicionalmente, en el CEDI la magnitud de las distancias que se recorren respecto al transporte y distribución son muy bajas porque la operación se ejecuta en un espacio cerrado.

- $\quad K$ vehículos o EMM heterogéneos de diferentes capacidades de carga (criterio 3) y K EMM heterogéneos de diferentes (dif) velocidades y manejo en altura (altura) (criterio 4)

En los 19 artículos evaluados, se modelaron K vehículos de diferentes capacidades de carga (criterio 3), pero en ninguno se representaron EMM con diversas velocidades de desplazamiento y manejo de productos en altura (criterio 4), lo cual, puede considerarse una limitación para modelar apropiadamente la conformación de lotes con ruteo la preparación de pedidos. La importancia de este criterio cuatro, se justifica en el contexto del CEDI, porque la velocidad de un EMM $k$ $\mathrm{K}$, tiene impacto en el tiempo de desplazamiento y la recogida de los $n$ productos i $\mathrm{OC}$ de $\mathrm{m}$ posiciones de almacenamiento j, lo cual afecta directamente el tiempo total y los costos de la operación. Por su parte, la capacidad de manipulación de productos en altura, es una restricción crítica que impacta en la programación y generación de lotes factibles, ya que en la preparación de pedidos se puede requerir la ejecución de movimientos verticales que permitan recoger $n$ productos i almacenados en posiciones de almacenamiento j, ubicadas en un segundo nivel o altura superior. Por esta razón, hace necesario asignar EMM k K, que tengan la capacidad técnica de ejecutar la operación.

\section{- $\quad n$ productos(prod) $\mathrm{i}^{€} \mathrm{OC}$ (criterio 5)}

En el SLR, se identificaron únicamente tres artículos, que modelaron múltiples productos i de diferentes pesos y volúmenes, en el problema HVRP. Los productos se representaron principalmente para controlar la capacidad de carga de los vehículos $k^{€} \mathrm{~K}$, asignados a las rutas, para garantizar la factibilidad de las soluciones (Rekersbrink et al., 2009; De la Cruz et al., 2013; Zhang et al., 2013).

En la conformación de lotes con ruteo, en la preparación de pedidos, los $n$ productos i€ OC, no sólo deberían controlar la capacidad de carga de los EMM 
$k^{€} K$ asignados, sino que también, deben restringir la recogida de las órdenes, teniendo en cuenta criterios operacionales como: contaminación cruzada, rotación de los $\mathrm{n}$ productos $\mathrm{i}$, disponibilidad del inventario, entre otros. Por los motivos expuestos, el modelado de $\mathrm{n}$ productos $\mathrm{i}$, impacta en la formulación de la función objetivo, restricciones, y estructura de los vecindarios de búsqueda en la conformación de lotes con ruteo para esta operación.

\section{- $\quad m$ posiciones (pos) de almacenamiento $\mathrm{j}$ (criterio 6)}

En 17 de los 19 artículos estudiados, se modelaron redes de transporte, distribución o procesos logísticos combinatorios con $\mathrm{N}$ clientes o nodos. En el contexto del CEDI, los $\mathrm{N}$ nodos equivalen a las $\mathrm{m}$ posiciones de almacenamiento $\mathrm{j}$, que guardan los $\mathrm{n}$ productos $\mathrm{i}$ que deben recoger para atender los requerimientos de los clientes. Por este motivo, las $\mathrm{m}$ posiciones de almacenamiento j son asimiladas como los $\mathrm{N}$ nodos en las redes de transporte y distribución.

Por estos motivos, en la conformación de lotes con ruteo, se deben representar redes de $\mathrm{N}$ nodos o m posiciones de almacenamiento j de tamaño genérico, que generen la flexibilidad del CEDI con diferentes dimensiones y capacidades de almacenamiento.

\section{- $\quad$ Restricción entre $\mathrm{n}$ productos $\mathrm{i}, \mathrm{m}$ posi- ciones j y K EMM (criterio 7)}

En siete de los artículos evaluados, los autores establecieron la restricción, que un vehículo $\mathrm{k} K$ únicamente podía visitar a un sólo cliente en la red de distribución, debido a las altas distancias entre los nodos o puntos de entrega en la red de distribución (Ceschia et al., 2010; Gendreau et al., 1999; Prado y Lopes, 2007; Euchi y Chabchoub, 2010; Prins, 2002; Leung et al.,2013; Kritikos y loannou, 2013). Incluso, en el artículo de Ceschia et al., (2010), no sólo se estableció la limitación operativa descrita anteriormente, sino que también se restringía, el tipo de productos i y prioridad de las órdenes de los clientes en la configuración de las rutas, con el fin de garantizar el cumplimiento de los tiempos de entrega.

En la conformación de lotes con ruteo, en la preparación de pedidos a diferencia del problema HVRP, que se orienta a procesos de transporte y distribución; se hace necesario, el modelado de una restricción, que determine y controle que EMM $k^{\epsilon} \mathrm{K}$, se puede asignar para la recogida de $\mathrm{n}$ productos $\mathrm{i}$ ubicados en las $\mathrm{m}$ posiciones de almacenamiento $\mathrm{j}$. En la preparación de pedidos, esta restricción tiene mayor relevancia que en los procesos de transporte y distribución modelados como un problema HVRP. Esto se plantea, porque los EMM $\mathrm{K}$ deben ejecutar desplazamientos horizontales y verticales, para recoger productos de posiciones de almacenamiento j ubicadas en segundos niveles de altura o superiores. Por esta razón, se hace necesario asignar EMM, que tengan la capacidad operativa para realizar la operación. En el transporte y distribución, los camiones o vehículos asignados sólo deben realizar desplazamientos horizontales en la red geográfi$\mathrm{ca}$, para atender los requerimientos de los clientes.

\section{- $\quad$ Tiempo de programación (criterio 8) y ma- nipulación (criterio 9) de los lotes con ruteo}

En ninguno de los 19 artículos evaluados, se modelaron los tiempos de programación (criterio 9), ni de manipulación de los $\mathrm{n}$ productos $\mathrm{i}$ asignados cada lote con ruteo, que se deben recoger de las $\mathrm{m}$ posiciones de almacenamiento $\mathrm{j}$, empleando los EMM heterogéneos $k^{€} K$ programados (criterio 10). La inclusión de estos tiempos, son determinantes en la conformación de lotes con ruteo factibles de allí, la importancia de su representación.

En la conformación de lotes con ruteo, en la preparación de pedidos, los tiempos de manipulación consideran la búsqueda de las $\mathrm{m}$ posiciones de almacenamiento j a visitar, la extracción de la cantidad de $n$ productos i OC y su descarga en el deposito (depot), usando los EMM heterogéneos $\mathrm{K}$ asignados. Adicionalmente, los tiempos de manipulación a modelar en la función objetivo, deben ser unitarios y variables, ya que estos dependen de las características técnicas de cada uno de los n productos i OC a recoger, las $\mathrm{m}$ posiciones de almacenamiento j a visitar (pasillo y altura del nivel), y los EMM k K programados. Con este enfoque, se contribuiría al estado del arte ya que los artículos relacionados con conformación de lotes con ruteo, modelaban el tiempo de manipulación como un valor fijo. Este supuesto puede conllevar a obtener soluciones no válidas, cuando en el CEDI, se tienen $\mathrm{K}$ $\mathrm{EMM}$ heterogéneos, $\mathrm{n}$ productos i de diversas características técnicas y $\mathrm{m}$ posiciones de almacenamiento $\mathrm{j}$ ubicadas en diferentes niveles de altura.

El tiempo de programación de cada lote con ruteo del conjunto conformado, también debería ser unitario y variable como el tiempo de manipulación, debido a que estos se calculan dependiendo de la cantidad de $n$ productos $i^{€} O C$ a recolectar, las ubicaciones de las $\mathrm{m}$ posiciones de almacenamiento $\mathrm{j}$ a visitar, las características de los equipos $k^{€} \mathrm{~K}$ asignados. De esta manera no sólo, se contribuye a la conformación de lotes con ruteo factibles, sino que también se aportaría al estado del arte, debido a lo novedoso de este enfoque en la literatura. 


\section{Disponibilidad del inventario de los $\mathbf{n}$ pro- ductos a recoger en el CEDI(Criterio 11)}

Ninguno de los 19 artículos evaluados, modeló la verificación de la disponibilidad del inventario de los $\mathrm{n}$ productos $i € \mathrm{OC}$, en el problema HVRP, que permitía configurar rutas de mínimo coste en el transporte, dis-tribución y otros procesos logísticos combinatorios. Por su parte, en la preparación, esta restricción es crítica, ya que sólo se pueden programar para conformación de lotes con ruteo, productos $i €$ OC con inventario disponible en el CEDI. Por los motivos expuestos, esta verificación tiene su impacto en la factibilidad de sus soluciones. Por los motivos expuestos, se justifica la necesidad de desarrollar un problema HVRP modificado, que incluya la restricción del inventario, en el modelamiento de la conformación de lotes con ruteo en la preparación de pedidos.

\section{Síntesis del análisis compa- rativo de la conformación de lotes con ruteo, en la prepa- ración de pedidos respecto al problema HVRP.}

A continuación, se sintetizan y cuantifican los resultados del análisis comparativo de los criterios de la conformación de lotes con ruteo, en la preparación de pedidos con relación al problema HVRP (...Tabla 3 ...).Estos resultados, no solo permiten cuantificar las diferencias y detectadas entre los problemas evaluados, sino también establecer las oportunidades de investigación y el aporte, que se pueden generar en la conformación de lotes con ruteo, en la preparación de pedidos. La estructura de la Tabla 3, incluye el criterio evaluado, y el número de artículos que cumplen total, parcial o no modelaron, el correspondiente criterio analizado.

Tabla 3. Síntesis y cuantificación de los criterios evaluados en el análisis comparativo.

\begin{tabular}{|c|c|c|c|c|c|}
\hline \multirow[t]{2}{*}{ Criterio evaluado } & \multicolumn{5}{|c|}{ Cuantificación del análisis comparativo } \\
\hline & Si & $\begin{array}{l}\text { \# de artícu- } \\
\text { los }\end{array}$ & Parcial & $\begin{array}{l}\text { \# de artícu- } \\
\text { los }\end{array}$ & No \\
\hline Conformación de lotes (order batching) (1) & & & & & $\mathrm{x}$ \\
\hline Ruteo (2) & & & $\mathrm{X}$ & 19 & \\
\hline $\begin{array}{l}\text { K equipos heterogéneos de diferentes capaci- } \\
\text { dades (3) }\end{array}$ & & & $x$ & 19 & \\
\hline $\begin{array}{l}\text { K equipos de diferentes velocidades y recogi- } \\
\text { da de productos en altura (4) }\end{array}$ & & & $x$ & 19 & \\
\hline n productos i (5) & & & $\mathrm{x}$ & 3 & \\
\hline m posiciones de almacenamiento j (6) & $\mathrm{X}$ & 19 & & & \\
\hline $\begin{array}{l}\text { Restricción de acceso a m posiciones j, } \\
\text { según él producto i y el EMM K (7) }\end{array}$ & & & & & $\mathrm{X}$ \\
\hline Tiempo de programación de cada lote (8) & & & & & $\mathrm{x}$ \\
\hline $\begin{array}{l}\text { Tiempo de extraer un producto i de la pos } j \\
\text { empleando el equipo } k \in K(9)\end{array}$ & & & & & $\mathrm{X}$ \\
\hline $\begin{array}{l}\text { Disponibilidad del inventario de los } n \text { produc- } \\
\text { tos } \mathrm{i}(10)\end{array}$ & & & & & $\mathrm{X}$ \\
\hline Totales & & $\%)$ & & $0 \%)$ & $5(50 \%)$ \\
\hline
\end{tabular}


De la cuantificación de las diferencias (Tabla 3), se detecta que cuatro $(40 \%)$ de los criterios evaluados deberían ser adaptados y otros cinco (50\%) deben incluirse en el problema HVRP, para que este pueda modelar el problema de conformación de lotes con ruteo, en la preparación de pedidos.

A continuación, se describen las adaptaciones e inclusión de nuevos componentes o restricciones, que se deberían desarrollar en el problema HVRP, con el fin de representar la conformación de lotes con ruteo, en la preparación de pedidos. Inclusive, las modificaciones son consideradas como oportunidades de investigación en esta línea del conocimiento:

- Integrar al ruteo un componente de conformación de lotes (criterio 2). Ninguno de los artículos del problema HVRP ninguno modela este componente, ni su integración con el ruteo (criterio 1), de allí la relevancia de esta diferencia.

- $\quad$ Modelar K equipos heterogéneos de diferentes capacidades de carga, velocidades y manejo de productos en altura. El $100 \%$ de los artículos del HVRP, representaron equipos de diferentes capacidades de carga (criterio 3), pero ninguno modeló la heterogeneidad de los equipos con diferencias en la velocidad y manipulación de productos en altura (criterio 4).

- Modelar una restricción, que controle la interacción entre los EMM heterogéneo $k \in K$, los $n$ productos $\mathrm{i}$, y las $\mathrm{m}$ posiciones de almacenamiento j. Esta restricción no fue detectada en ninguno de los artículos HVRP, y puede ser considerada determinante en la validez de la conformación de lotes con ruteo, en la preparación de pedidos (criterio 7).

- $\quad$ Representar el tiempo de programación y manipulación unitario que debe depender de la cantidad de productos i OC a recoger, las posiciones de almacenamiento j a visitar, y las características técnicas de los K EMM asignados(criterios 8 y 9). En ninguno de los artículos evaluados del problema, se modelaron estos criterios, los cuales suelen ser críticos en la conformación de lotes con ruteo.

- Verificar disponibilidad del inventario de los $n$ productos i OC a preparar, incluyendo la integración con Tecnologías de la Información y Comunicaciones (TIC) como el Warehouse Management System (WMS), que facilite la gestión de la información (10). Ninguno de los artículos revisados del problema HVRP implementó este criterio.

Adicionalmente, debe resaltarse que el criterio 6, es el único que no requiere modificación, ya que las $\mathrm{m}$ posiciones de almacenamiento j, en la conformación de lotes con ruteo, en la preparación de pedidos, son equivalentes a los $\mathrm{N}$ nodos de la red de transporte y distribución modelados en los artículos del problema HVRP.

Finalmente, del análisis comparativo desarrollado, se detecta la necesidad de modelar un problema de conformación de lotes con ruteo de mínimo tiempo, en la preparación de pedidos, que tenga en cuenta $\mathrm{K}$ equipos heterogéneos (diferentes velocidades, capacidades y manejo de altura), $\mathrm{n}$ productos $\mathrm{i}, \mathrm{m}$ posiciones de almacenamiento j, la verificación de disponibilidad del inventario y demás restricciones descritas. Con este enfoque, se cubriría el vacío detectado en el arte del arte, y aportar al avance del conocimiento en el modelado de la preparación de pedidos.

\section{Conclusiones}

Del desarrollo del artículo, se detectó la importancia de modelar K EMM heterogéneos en la conformación de lotes con ruteo, en la preparación de pedidos, debido a que en los CEDI con sistemas de estanterías, los productos $i € O C$ deben recogerse de $m$ posiciones de almacenamiento j ubicados en diferentes niveles de altura. Debe resaltarse, que en el estado del arte del problema de conformación de lotes con ruteo, para esta operación, únicamente se modelaba un EMM de capacidad infinita o fija, o K EMM homogéneos. Con relación al análisis comparativo respecto al problema HVRP, no se identificaron artículos que abordaran la conformación de lotes con ruteo en la preparación de pedidos, sino que se enfocaban principalmente en la configuración de rutas de mínimo coste en el transporte y la distribución. Por estos motivos, a partir de un análisis comparativo detallado de 10 criterios, se iden-tificó la oportunidad de investigación modelar un problema de conformación de lotes con ruteo de mínimo tiempo, en la preparación de pedidos, que considere $\mathrm{K}$ equipos heterogéneos, $n$ productos $i, m$ posiciones de almacenamiento j, la verificación de disponibilidad del inventario y otras restricciones. Además, debe indicarse que sólo uno de los criterios comparados denominado $\mathrm{m}$ nodos $\mathrm{o}$ posiciones de almacenamiento j, en el problema HVRP no requería modificaciones para representar la conformación de lotes con ruteo, en la preparación de pedidos. En tanto los otros nueve criterios debían ser adaptados o incluidos. De esta manera, se establecieron las diferencias, y se detectó la oportunidad de investigación de desarrollar un problema de conformación de lotes con ruteo, en la preparación de pedidos, el cual, se puede convertir en un aporte al conocimiento en esta línea de investigación. 
Agradecimientos: Este artículo es resultado de la tesis de doctorado: "Metaheurísticos para solucionar problemas de conformación de lotes con ruteo, en el acomodo y la preparación de pedidos, considerando $\mathrm{K}$ equipos heterogéneos", en la Universidad Nacional de Colombia, Sede Medellín y la pasantía de investigación desarrollada en la Universidad de Sevilla.

\section{Referencias}

AlBAREDA, M., ALONSO, A., MOLINA, E. y DE BLAS, C. (2009). "Variable Neighborhood Search for Order Batching In A Warehouse ». Asia-Pacific Journal of Operational Research (APJOR), 26(05), 655-683.

BARTHOLDI, J., y HACKMAN, S. (2011). Warehouse \& Distribution Science: The Supply Chain and Logistics Institute.

BRANDÃO, J. (2009). «A deterministic tabu search algorithm for the fleet size and mix vehicle routing problem ». European Journal of Operational Research, 195(3), 716-728.

BRANDÃO, J. (2011). «A tabu search algorithm for the heterogeneous fixed fleet vehicle routing problem ». Computers \& Operations Research, 38(1), 140-151.

CESCHIA, S., GASPERO, L., y SCHAERF, A. (2011). «Tabu search techniques for the heterogeneous vehicle routing problem with time windows and carrier-dependent costs». Journal of Scheduling, 14(6), 601-615.

CHOI, E., y TCHA, D. (2007). «A column generation approach to the heterogeneous fleet vehicle routing problem ॥. Computers \& Operations Re-search, 34(7), 2080-2095.

DANIELS, R., RUMMEL, J. y SCHANTZ, R. (1998). «A model for warehouse order picking». European Journal of Operational Research, 105(1), 1-17.

DHARMAPRIYA, U., y KULATUNGA, A. (2011). «New strategy for warehouse optimization-lean warehousing ». En Proceedings of the 2011 International Conference on Industrial Engineering and Operations Management. Council of Supply Chain Management Professionals.

DE KOSTER, M., VAN DER POORT, S., y WOLTERS, M. (1999). «Efficient order batching methods in warehouses ». International Journal of Production Research, 37(7), 1479-1504.
DE LA CRUZ, J., PATERNINA, C., CANTILLO, V., y MONTOYA, J. (2013). «A two-pheromone trail ant colony system-tabu search approach for the heterogeneous vehicle routing problem with time windows and multiple products ». Journal of Heuristics, 19(2), 233-252.

DUHAMEL, C., LACOMME, P., y PRODHON, C. (2012). «A hybrid evolutionary local search with depth first search split procedure for the heterogeneous vehicle routing problems». Engineering Applications of Artificial Intelligence, 25(2), 345-358.

EUCHI, J., y CHABCHOUB, H. (2010). «A hybrid tabu search to solve the heterogeneous fixed fleet vehicle routing problem ». Logistics Research, 2(1), 3-11.

GENDREAU, M., LAPORTE, G., y YELLE, S. (1997). «Efficient Routing of Service Vehicles». Engineering Optimization, 28(4), 263-271.

HENN, S. (2012). «Algorithms for on-line order batching in an order picking warehouse». Computers \& Operations Research, 39(11), 2549-2563.

HENN, S. y SCHMID, V. (2013). «Metaheuristics for order batching and sequencing in manual order picking systems ». Computers \& Industrial Engineering, 66(2), 338-351.

HENN, S. y WÄSCHER, G. (2012). «Tabu search heuristics for the order batching problem in manual order picking systems». European Journal of Operational Research, 222(3), 484-494.

HENN, S., KOCH, S., DOERNER, K., STRAUSS, C., y WÄSCHER, G. (2010). "Metaheuristics for the order batching problem in manual order picking systems». BuR Business Research Journal, 3(1), 1-24.

HO, Y., SU, T. y SHI , Z. (2008). «Order-batching methods for an order-picking warehouse with two cross aisles». Computers \& Industrial Engineering, 55, 321-347.

IMRAN, A., SALHI, S. y WASSAN, N. (2009). "A variable neighborhood-based heuristic for the heterogeneous fleet vehicle routing problem». European Journal of Operational Research, 197(2), 509-518.

KITCHENHAM, B. (2004). Procedures for performing systematic reviews. Keele, UK, Keele University, 33, 2004. 
KRITIKOS, M. y IOANNOU, G. (2013). «The heterogeneous fleet vehicle routing problem with overloads and time windows $»$. International Journal of Production Economics, 144(1), 68-75.

LEUNG, S., ZHANG, Z., ZHANG, D., HUA X y LIM, M. (2013). «A meta-heuristic algorithm for heterogeneous fleet vehicle routing problems with two-dimensional loading constraints». European Journal of Operational Research, 225(2), 199-210.

LI, X., LEUNG, S. y TIAN, P. (2012). «A multistart adaptive memory-based tabu search algorithm for the heterogeneous fixed fleet open vehicle routing problem». Expert Systems with Applications, 39(1), 365-374.

MANZINI, R., GAMBERI, M. y REGATTIERI, A. (2006). «Design and control of an AS/RS». The International Journal of Advanced Manufacturing Technology, 28(7), 766-774.

MATUSIAK, M., DE KOSTER, R., KROON, L., y SAARINEN, J. (2014). "A fast simulated annealing method for batching precedence-constrained customer orders in a warehouse». European Journal of Operational Research, 236(3), 968-977.

OZFIRAT, P. y OZKARAHAN, I. (2010). «A Constraint Programming Heuristic for a Heterogeneous Vehicle Routing Problem with Split Deliveries». Applied Artificial Intelligence, 24(4), 277-294.

PENNA, P., SUBRAMANIAN, A. y OCHI, L. (2013). «An Iterated Local Search heuristic for the Heterogeneous Fleet Vehicle Routing Problem». Journal of Heuristics, 19(2), 201-232.
PRINS, C. (2002). «Efficient Heuristics for the Heterogeneous Fleet Multitrip VRP with Application to a Large-Scale Real Case». Journal of Mathematical Modelling and Algorithms, 1(2), 135-150.

PRINS, C. (2009). «Two memetic algorithms for heterogeneous fleet vehicle routing problems». Engi-neering Applications of Artificial Intelligence, 22(6), 916-928.

REKERSBRINK, H., MAKUSCHEWITZ, T. y SCHOLZ, B. (2009). «A distributed routing concept for vehicle routing problems». Logistics Research, 1(1), 45-52.

RUBRICO, J., HIGASHI, T., TAMURA, H. y OTA, J. (2011). «Online rescheduling of multiple picking agents for warehouse management». Robotics and Computer-Integrated Manufacturing, 27(1), 62-71.

SUBRAMANIAN, A., PENNA, P., UCHOA, E., y OCHI, L. (2012). «A hybrid algorithm for the heterogeneous fleet vehicle routing problem». European Journal of Operational Research, 221(2), 285-295.

TSAI, C., LIOU, J. y HUANG, T. (2008). «Using a multiple-GA - method to solve the batch picking problem: considering travel distance and order due time». International Journal of Production Research, 46(22), 65-33. 